\title{
Behind every innovative solution lies an obscure feature
}

\author{
Tony McCaffrey* \\ Cognitive Psychology \\ University of Massachusetts Amherst, USA \\ E-mail: amccaffr@psych.umass.edu
}

\section{Lee Spector}

\author{
Cognitive Science \\ Hampshire College, USA \\ E-mail: 1spector@hampshire.edu
}

*Corresponding author

\begin{abstract}
The Obscure Features Hypothesis (OFH) for innovation states that a two-step process undergirds almost all innovative solutions: (1) notice an infrequently observed or new (i.e., obscure) feature of the problem and (2) construct an interaction involving the obscure feature that produces the desired effects to solve the problem. The OFH leads to a systematic derivation of innovation-enhancing techniques by engaging in two tasks. First, we developed a 32-category system of the types of features possessable by a physical object or material. This Feature Type Taxonomy (FTT) provides a panoramic view of the space of features and assists in searches for the obscure ones. Second, we are articulating the many cognitive reasons that obscure features are overlooked and are developing countering techniques for each known reason. We present the implications and techniques of the $\mathrm{OFH}$, as well as indicate how software can assist innovators in the effective use of these innovation-enhancing techniques.
\end{abstract}

Keywords: Innovation; Semi-automated creativity; Problem solving

Biographical notes: Tony McCaffrey is currently a postdoctoral fellow at the University of Massachusetts Amherst conducting research with the NSF Center for e-Design. He received his Ph.D. in cognitive psychology from the University of Massachusetts Amherst.

Lee Spector is a Professor of Computer Science in the School of Cognitive Science at Hampshire College. He received his Ph.D. in computer science at the University of Maryland.

\section{Introduction}

An examination of all the innovation problems used in psychology experiments (i.e., insight problems) and 1,001 historic inventions listed in Challoner (2009) suggests that at some point in the innovation process an obscure feature of the problem was noticed and used as the basis of a novel solution. As a simple illustrative example of a problem requiring innovation, we use an insight problem: the Two Rings Problem (McCaffrey, 
2012). Suppose you need to fasten together two weighty steel rings in a figure-eight configuration using only a long candle, a match, and a two-inch cube of steel. A bond created with melted wax is not strong enough. However, if one notices that the wick is a string that can be extricated by scraping away the wax on the steel cube, then the rings can be tied together and the problem solved. The fact that the wick is a string is a frequently overlooked feature of this problem. Once noticed then the problem becomes easy for people to solve.

Logically speaking, if an unsolved problem is solvable, then something crucial to the solution is being overlooked. If it is overlooked by the problem solving community for a long period of time, then the crucial something is either infrequently-noticed or perhaps never-before noticed (i.e., obscure). We will use the term features to name all the possible things that could be overlooked about a problem. Systematically characterizing the many types of features possessable by a problem will help give us a panoramic view of the feature space and assist us in searching this space. Articulating the many cognitive reasons why we overlook obscure features allows us to devise countering techniques so that obscure features are more easily uncovered. In general, the more obscure features we can uncover about the problem, the higher the probability of uncovering the key obscure feature that a solution is based upon. In this article, we begin to characterize the feature space, name cognitive obstacles to innovation, and devise countering techniques for them.

\section{Three locations of obscure features}

We view the innovative problem solving process as two networks growing toward each other. The goal is a top-down network that grows downward as the expression of the goal is refined. A bottom-up network presents the available objects and materials and grows upward as more of their features are uncovered. Quite often, as in the Two Rings Problem, the uncovering of a single feature - that the wick is a string - is sufficient to bridge the gap between the two networks. As illustrated in Fig. 1, the uncovering of a single feature may suddenly connect the two networks in an aha moment. More fully, however, the middle part of the diagram needs to be fleshed out with interactions among the available objects/materials that produce the needed effects. In our example, the candle wax interacts with the steel cube in a scraping manner in order to free the wick. The wick interacts with the two steel rings by tying them together in order to fasten them. For simple problems such as the Two Rings Problem, the interactions in the middle part of the diagram rarely need to be filled out explicitly.

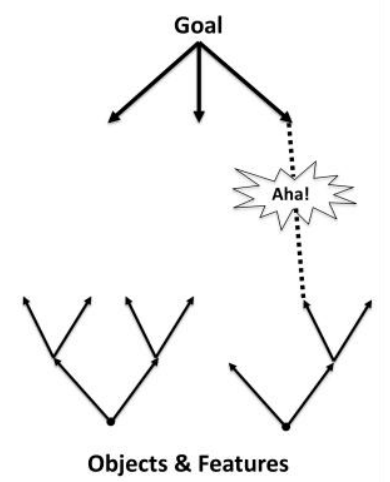

Fig. 1. The two-network view of innovation 
Overall, a goal is a set of desired effects. The objects/materials are the substances that can cause effects during interactions. If a series of interactions can be devised among the available objects/materials that produce all the desired effects, then the problem is solved. Given this framework for innovative problem solving, we can point to three areas of the diagram where obscure features might reside: in the expression of the goal, among the features of the objects/materials, and within the effects produced by interactions. An interaction might produce effects that are overlooked.

In this article, we focus on the first two locations. Future research will explore uncovering obscure effects of interactions. Below, we first look at several other approaches to innovation in psychology and engineering. Second, we present a systematic approach to uncovering obscure features of physical objects/materials. Third, we turn to the ways that goals can hide obscure features that can be crucial to solving innovation problems.

\section{Previous theories of innovation}

There are two over-arching theories of innovation in cognitive psychology. First, the Representation Change View (Knoblich, Ohlsson, Raney, Haider, \& Rhenius, 1999; Ohlsson, 1992) states that people initially adopt an incorrect or incomplete representation of the problem and must change their representation in order to solve the problem. The Representation Change View has not led to a systematic way to devise techniques to improve innovation because it has not answered the following questions. What are the many types of representation change? How can we assist humans to notice the overlooked types? In our view, it would be a daunting task to attempt to list out all possible representation changes. For this reason, we are sceptical as to whether the Representation Change View could be made into a systematic approach. Second, the Distant Association View (Mednick, 1962) states that innovative solutions rely on associations that are distant from the original concepts used in the problem. The Distant Association View also has not led to a systematic research program because it has not answered the same types of questions. What are the types of associations? How can we assist humans to notice the distant members or each type? How distant from the original concept are the most promising associations? In our view, listing out the types of associations seems to be a more tractable problem. In fact, because an association and a feature are closely related concepts, the $\mathrm{OFH}$ approach can be viewed as a way of systematizing the Distant Association View. After characterizing the many types of features of a physical object/material, we then attempt to devise a technique to uncover the obscure members of each type. In this way, we consider our approach to be systematic.

From the engineering field, TRIZ is a systematic approach to innovation based on contradictions (Altshuller, 1996). A contradiction occurs when two seemingly incompatible things are desired (e.g., better gas mileage and greater acceleration). Altshuller (1996) articulated forty principles that can help overcome contradictions. Given a contradiction, look up the two contradictory demands in a TRIZ table, which will suggest several principles that have helped overcome this type of contradiction in the past. Although helpful for problems that contain contradictions, not all problems involve contradictions. Suppose you are given the goal of designing a new type of candle for a candle manufacturer. There is no underlying contradiction preventing you from designing a new candle. From the perspective of the OFH approach, as we will demonstrate below, you are simply overlooking an obscure feature of a candle that could become the basis of a new design. Further, even for problems containing contradictions, the key to the 
solution is to locate the key obscure feature(s) that form the basis of the solution. In this sense, the OFH approach has the possibility of being complementary to TRIZ. While TRIZ helps articulate the contradiction, the OFH approach helps uncover the obscure features that the solution will be based upon. In sum, whether a problem requiring innovation contains a contradiction or not, the solution will be based on an at least one obscure feature of the problem.

\section{Obscure features of objects/materials}

In the next sections, we focus on unearthing obscure features of the objects and materials of a problem. Specifically, we present two well-known cognitive obstacles to innovation: design fixation (Jansson \& Smith, 1991) and functional fixedness (Duncker, 1945). We then analyze them from the perspective of the OFH and present countering techniques.

\subsection{Design fixation}

Design Fixation is the tendency to fixate on the features of known solutions when trying to create novel solutions (Jansson \& Smith, 1991). To alleviate this fixation, we propose to construct a panoramic view of the possible types of features. In this way, innovators can see the obscure feature types available for new designs as well as the feature types that previous solutions have been built upon. To create our category system of possible feature types, we re-examined our collection of more than 1,000 problems and noted that the key obscure features needed for a solution all fell into one of 32 types of features. This category system of feature types contains obvious features (e.g., shape, size, and material composition) as well as many less obvious feature types. Our current Feature Type Taxonomy (FTT) is extensive, but we continue to test and refine it. Presently, the full category system is proprietary until the release of our software, which relies upon it. Consequently, we cannot present the full FTT in this article, but will present all the feature types needed to understand our examples.

To measure how many of the feature types are usually overlooked, we had fifteen subjects write down as many features and associations as they could in four minutes for each of a set of fourteen common objects (e.g., candle and broom). We classified their answers among the 32 feature types of our taxonomy. On average, subjects listed only one response or no responses for 20.7 of the 32 categories $(64.7 \%)$. Nearly two-thirds of the feature types for these common objects were either completely overlooked (no responses) or underexplored (only one response). If innovative solutions are built upon obscure features, then this result implies that many new designs for these common objects have yet to be created.

To test this hypothesis, we worked with the results from a candle, created as many new designs as we could in two one-hour sessions, obtained audiences with two candle companies, and asked them to assess the novelty of our designs.

Fig. 2 shows our results for a candle in the form of feature type spectrum (FTS), named as such because it gives a kind of spectral analysis to the features of a candle (McCaffrey \& Spector, 2011b). The y-axis of Fig. 2 represents the average number of times these subjects listed a feature of a particular type. The $\mathrm{x}$-axis shows the 32 feature types presented by number.

Fig. 2 shows a clear pattern of underexplored and ignored feature types that could become the basis for innovation. The low bars and non-existent bars of Fig. 2 point to the obscure feature types upon which to build new candle designs. Using Fig. 2, we were 
able to create ten new candle designs in two one-hours sessions. The two candle companies assessed that nine of the ten designs were indeed novel. Pilgrim Candle is licensing our self-snuffing candle described below and is interested in two of our other designs.

The self-snuffing candle was built upon two overlooked features. No one mentioned anything about the motion (type \#28) of a candle (e.g., candles are motionless when they burn) or weight (type \#9: candles lose weight when they burn). Using weight loss to try to generate vertical motion, we proceeded to interact our weight-losing candle with other objects/materials commonly associated with vertical motion. Searching for objects commonly associated with vertical motion reveals a list, which includes a justice scale, elevator, helicopter, kite, rocket, trampoline, and catapult. Using the first object in the list as an example, we placed a candle on one side of a scale-like structure and counterbalanced it with a weight on the other side. Just for fun, put a snuffer at the top so the candle eventually moves into the snuffer as it loses weight and extinguishes itself.

Candle: Feature Type Breakdown

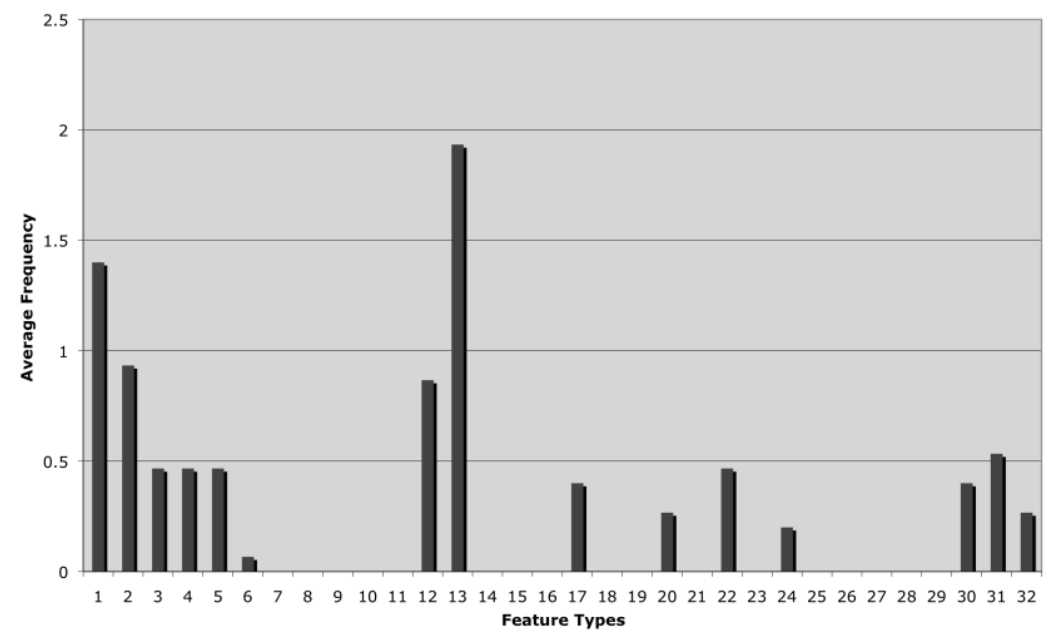

Fig. 2. A feature type spectrum for a candle

Candles have existed for approximately 5,000 years. Certainly, the space of candle designs has nearly been exhausted. However, our results point to the opposite conclusion. If novel candle designs are built upon obscure features and people overlook approximately 18 of the 32 types of features (56\%) of a candle (Fig. 2), then the space of new candle designs is possibly quite richly populated. Using the FTS method, novice candle designers could create nine novel designs in the space of two hours. The FTS allows innovators to focus on the overlooked feature types of an object, thus relieving design fixation which keeps innovators fixated on the feature types used in current designs.

\subsection{Computer assistance for the FTS}

We propose that the computer representation of each feature of an object include information as to its degree of commonality and its type of feature (e.g., shape or size). One possible way to collect this information is to use surveys (e.g., online) by having people list as many features and associations as they can. Then for each feature, two 
numbers can be stored in the representation: the number of people who listed the feature and the total number of people in the survey. In this way, future surveys can add to both numbers to keep a running tally of the overall commonality of that feature. Applications can then use different cutoffs in expressing what degree of response constitutes that a feature is commonly noticed.

\subsection{Functional fixedness}

Functional fixedness is traditionally characterized as the tendency to fixate on the common use of an object or one of its parts (Duncker, 1945). Until McCaffrey (2012), there has not been an effective way to counteract it. Analysis of all the problems used in psychology experiments that evoke functional fixedness (e.g., the Two Rings Problem), however, suggests a different characterization of functional fixedness that leads to a way to counteract it. To overcome functional fixedness requires unearthing obscure members of only four feature types: parts, shape, size, and material (McCaffrey, 2012).

To help people notice the overlooked parts, material, shape, and size, we devised the Generic Parts Technique (GPT), in which a participant creates a parts tree for an object (Fig. 3) in the following manner. For each description created for a part, a participant should ask, "Can this be decomposed further?" If so, they should break that part into its sub-parts and create another level of the hierarchy for the sub-parts. The second question to ask is "Does this description imply a use?" If so, they should create a more generic description based on material and shape. The result of this procedure for the candle from the Two Rings Problem is a tree diagram (Fig. 3) in which the description in each leaf does not imply a use and involves the material and shape of the part under consideration. Further, because smaller parts emerge as we progress down the tree toward the leaves, this process also brings attention to the size of each of the parts.

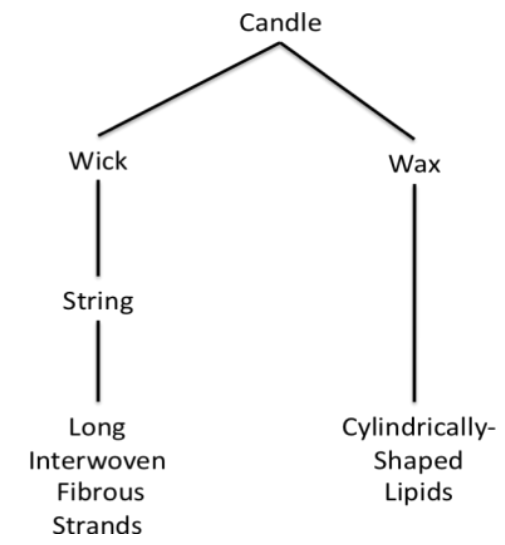

Fig. 3. Generic parts diagram for a candle

In this case, because the word wick implies a use (i.e., burning to emit light), we created a more generic description based on its material: string. Because string also implies a use (i.e., tying things together), we created a more generic description primarily involving shape and material: long, interwoven, fibrous strands.

To test the effectiveness of the GPT, fourteen subjects were trained in the use of the GPT and worked on eight insight problems - all of which suffered from functional fixedness. Their problem-solving performance was compared against a control group of 
fourteen subjects that were not trained in any technique. The GPT group solved $67 \%$ more problems than a control group. The GPT group had an $83 \%$ solution rate compared to a $49 \%$ solution rate for the control group: $t(26)=4.23, p<.001$ (McCaffrey, 2012).

\subsection{Computer assistance for the GPT}

Although subjects using the GPT solved significantly more problems than those who did not, still the GPT subjects did not solve $100 \%$ of the problems. Consequently, there is still room for improvement. Examining the paper sheets used by the GPT subjects suggests that the subjects were not rigorous when creating the parts diagrams. Most drawn diagrams only proceeded to the first level of the hierarchy. A post-problem questionnaire revealed that the majority of the subjects performed the GPT either entirely "in their heads," or partially on paper and partially "in their heads." Consequently, crucial obscure features may have remained concealed for certain problems. To attempt to alleviate this lack of rigor, we implemented the GPT in software. The hope was that using the GPT software might help subjects to more fully complete the parts diagrams.

Thus far, two pilot subjects used the GPT software on the eight insight problems used in the psychology experiment just described (McCaffrey \& Spector, 2011a). Both subjects solved all eight problems. The program helps users construct parts diagrams for objects by continually asking questions such as "Can this part be decomposed further into parts?", "What is the material make-up of this part?", and "Does your description imply a use?" These preliminary results suggest that software could help users more carefully construct parts diagrams, which could lead to better problem solving performance for innovation problems suffering from functional fixedness.

\subsection{Summary of obscure features of objects/materials}

In sum, we presented two cognitive obstacles to innovation, design fixation and functional fixedness, and presented countering techniques for them. We plan to continue to articulate new cognitive obstacles to innovation and devise their countering techniques. The FTT assists us in characterizing the feature types that are obscured by a cognitive obstacle and helps give us a panoramic of the many feature types possessable by a physical object or material.

\section{Obscure features of goals}

In the following sections, we explore two possible ways that the expression of the goal could hide information crucial to a solution. Hirtz, Stone, McAdams, Szykman, and Wood (2002) posits that all engineering operations and goals can be expressed by a verb (e.g., fasten rings together). In the case of an engineering operation, the verb expresses the effect that the operation enacted. In the case of an engineering goal, the verb expresses the desired effect. Following their lead, we will focus on two ways to unearth obscure features of verbs that could then assist with innovation. We introduce two new cognitive obstacles, narrow verb associations and assumption blindness, and their counteracting techniques. 


\subsection{Overcoming narrow verb associations}

Again, consider The Two Rings problem. Its goal is to fasten the rings together. The solution ends up being tying the rings together. The verb tie is a more specific version of the verb fasten. If problem solvers could list out the many specific versions of the goal verb fasten, then they could think of many concrete ways to fasten the rings together. Specifically, the online dictionary and thesaurus WordNet (Miller, 1995) developed at Princeton University lists 61 concrete ways to fasten things together: tie, glue, clip, buckle, weld, velcro, and many others. WordNet structures the synonyms of verbs into those that are more general (hypernyms) and those that are more specific (hyponyms). Fig. 4 shows a small portion of WordNet's hierarchy for the verb fasten.

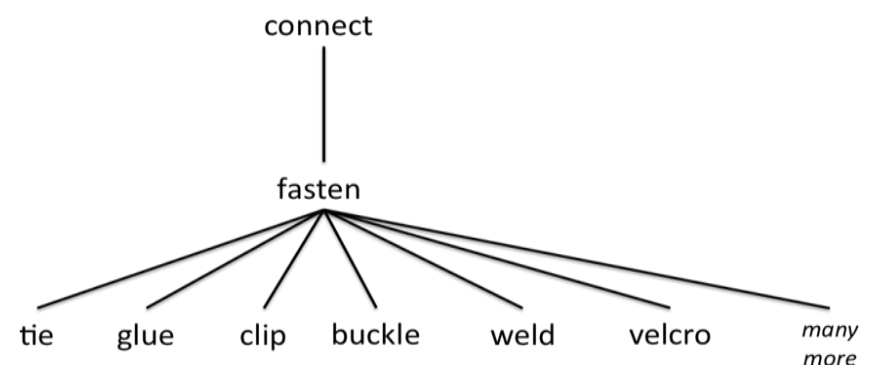

Fig. 4. Sample of verb hierarchy in WordNet

We suggest that WordNet's list of verb hyponyms could be used by problem solvers to help consider the many ways to enact a verb. To test the hypothesis that WordNet contains more synonyms than people can think of on their own, we had 15 subjects list all the synonyms they could for six verbs. The number of hyponyms in WordNet are given in parentheses after each verb: fasten (61), remove (172), guide (50), transport (46), mix (24), and separate (115). Subjects listed 8.1 synonyms on average with a margin of error of 2.8. Further, of the 8.1 synonyms listed only 3.9 were hyponyms. Given the vast number of hyponyms available in WordNet, it seems that WordNet could be a rich source of specific ways to enact more general goal verbs such as fasten or remove. In other words, the proper use of WordNet could help counteract our tendency for narrow verb associations.

\subsection{Computer assistance for narrow verb associations}

We propose to develop an engineering-specific version of the WordNet verb structure. Perhaps, there are verbs specific to engineering that should be added to the verb hierarchies that are currently in WordNet. Specifically, Hirtz et al. (2002) proposes a hierarchy of approximately 200 verbs that are important for expressing operations and goal in engineering. Merging the hierarchies in WordNet and Hirtz et al. (2002) could provide a helpful resource for problem solvers. Problem solvers could move up and down the verb hierarchy in search of the best way to articulate their goal as well as peruse the many ways that a goal could be concretely accomplished.

\subsection{Overcoming assumption blindness}

Navigating through a verb hierarchy such as in Fig. 4 may be insufficient at times to helping innovators find the verb that best expresses a possible solution. The reason is that each verb hides many assumptions about the features that a solution using that verb 
would possess. For example, an engineering firm presented us with the unsolved problem: "adhere a coating to Teflon." Teflon is a no-stick surface and very aptly named. The verb used to express the goal is not innocent but channels the human mind to consider certain kinds of solutions and ignore others. We call this channeling assumption blindness because we often are blind to the restrictive or even misleading assumptions that are hidden behind the chosen goal verb.

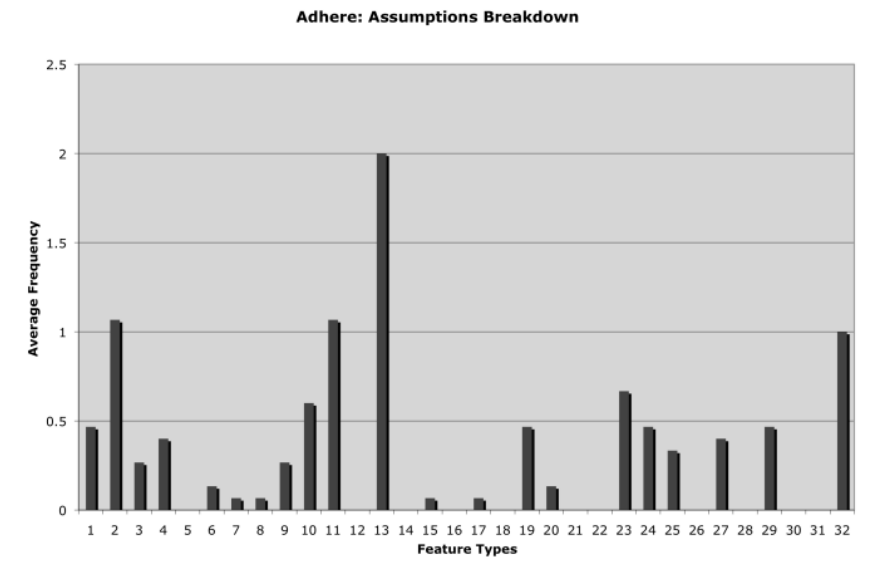

Fig. 5. Features assumed by the verb adhere

To begin to measure the range of assumptions that people are aware of, we asked 15 subjects to list all the assumptions they could for the verb adhere. Specifically, we asked them to consider what features of the final solution they were assuming when they used the verb adhere. We then classified the assumptions based on our FTT to get a sense of all the features of the final solution that subjects tend to be aware of. Based on the pattern of Fig. 5, people are generally unaware of $47 \%$ (15 out of 32) of the feature types assumed by the verb adhere. Fifteen out of the 32 features types listed either one or no responses. For example, subjects were aware that the verb adhere implied that a chemical process would be used (i.e., type of energy) and that two things were being adhered together (i.e., number). However, no subject mentioned the key to solving this unsolved problem: namely, the verb adhere assumes direct contact between the things being adhered together (i.e., spatial relation). Negating all three of these assumptions simultaneously permitted us to create a solution deemed plausible by a large chemical engineering firm. This process led to the idea of sticking a coating "through" Teflon to a magnetic surface beneath the Teflon (i.e., a sandwich of three surfaces in which the coating indirectly sticks to the Teflon due to its attraction to the magnetic surface). Of course, the coating would need to possess the proper make-up in order to induce sticking. This plausible solution required negating three assumptions in order to simultaneously consider a different type of energy connecting a different number of entities in a different spatial configuration. In general, the more assumptions we are aware of for the chosen goal verb, the more effectively we can explore the space of possible solutions.

In sum, the choice of the goal verb contains many assumptions of the features that the final solution will possess. The FTT provides a panoramic lens to observe a wide range of feature types assumed by our verb choice. When listing out these assumed features, our subjects overlooked nearly half of the types of features possible. Any of these feature types could contain the key feature that, if negated, could become the crucial feature for solving an important design problem. 


\subsection{Computer assistance for assumption blindness}

Again, the taxonomy of Hirtz et al. (2002) states that almost all engineering goals can be expressed by one of approximately 200 verbs. Using the FTT we can create an extensive database of assumed features for each verb in the hierarchy and embed them in software. Then, when solving a problem, users of the software can examine and negate the rich set of features assumed by the verb used to describe their goal. Further, users can add new assumed features to the current lists in the software. In addition, users can position new verbs in the software's taxonomy. In our Teflon example, the verb adhere is not in the taxonomy of Hirtz et al. (2002) but can be added in its proper place as adhere is a more specific synonym of the verb connect. The verb adhere will inherit some of the features assumed by the verb connect as well as take on specific features not attributable to the verb connect.

\subsection{Summary of obscure features of goals}

Currently, we have focused upon the verb of a goal and have dealt with two ways that the verb can hide things that could be important in innovative problem solving: narrow verb associations and assumption blindness. Future research will seek out other ways that the goal verb obscure features relevant to innovative solutions. Further, because there is more to a goal than just its verb (e.g., fasten rings together), we are also investigating the other parts of an articulated goal in terms of what features it might be obscuring.

\section{A toolkit of innovation-enhancing techniques}

Obscure features are critical to innovation. If a solvable problem is currently unsolved, then at least one obscure feature is being overlooked. The OFH describes two steps underlying the solutions to the many problems we examined: find obscure features and construct interactions involving the unearthed obscure features. In this article, we focused on two sources of obscure features: the available objects/materials and the goal. Specifically, we articulated two cognitive obstacles from each source and devised their countering techniques. Where possible, we described how software could be constructed to guide designers in the effective use of these innovation-enhancing techniques. Further, we devised an extensive category system of possible feature types for an object/material (the FTT). Future work includes the following: articulating other cognitive obstacles and devising their countering techniques, continuing to test and refine the FTT, and beginning to analyze the third general source of obscure features: the obscure effects that emerge from an interaction among objects/materials. It is our belief that the OFH approach will continue to produce helpful techniques implementable in software that can improve human innovation.

\section{Acknowledgements}

This material is based upon work supported by the National Science Foundation (NSF) of the USA under Grant No. 1017817. Any opinions, findings, and conclusions or recommendations expressed in this publication are those of the authors and do not necessarily reflect the views of the NSF. 


\section{References}

Altshuller, G. S. (1996). And suddenly the inventor appeared: Triz, the theory of inventive problem solving. Technical Innovation Center, Worcester, MA. ISBN 0-96407402-8.

Challoner, J. (2009). 1,001 Inventions that changed the world. Hauppauge, NY: Barron's Educational Series.

Duncker, K. (1945). On problem-solving. Psychological Monographs, 58(5), Whole No. 270.

Hirtz, J., Stone, R., McAdams, D., Szykman, S., \& Wood, K. (2002). A functional basis for engineering design: Reconciling and evolving previous efforts. Res Eng Design, $13,65-82$.

Jansson, D. G., \& Smith, S. M. (1991). Design fixation. Design Studies, 12(1), 3-11.

Knoblich, G., Ohlsson, S., Raney, G. E., Haider, H., \& Rhenius, D. (1999). Constraint relaxation and chunk decomposition in insight problem solving. Journal of Experimental Psychology: Learning, Memory, and Cognition, 25, 1534-1555.

McCaffrey, T. (2012). Innovation relies on the obscure: A key to overcoming the classic functional fixedness problem. Psychological Science, 23(3), 215-218.

McCaffrey, T., \& Spector, L. (2011a). How the obscure features hypothesis leads to innovation assistant software. Proceedings of the Second International Conference on Computational Creativity, 120-122.

McCaffrey, T., \& Spector, L. (2011b). Innovation is built upon the obscure: Innovationenhancing software for uncovering the obscure. In A. K. Goel, F. Harrell, B. Magerko, Y. Nagai, \& J. Prophet (Eds.), Proceedings of the 8th ACM Conference on Creativity and Cognition (pp. 371-372). New York, NY: Association for Computing Machinery. doi:10.1145/2069618.2069701

Mednick, S. (1962). The associative basis of the creative process. Psychological Review, 69, 220-232.

Miller, G. A. (1995). WordNet: A lexical database for English. Communications of the ACM, 38(11), 39-41.

Ohlsson, S. (1992). Information processing explanations of insight and related phenomena. In M. T. Keane \& K. J. Gilhooly (Eds.), Advances in the psychology of thinking (pp. 1-44). London, England: Harvester Wheatsheaf. 